\title{
Teste de um protótipo limitador de corrente falta supercondutor de cerâmica SmBaCuO
}

\author{
C. A. C. Passos ${ }^{1 \mathrm{a}}$, Danilo P. e Silva ${ }^{2}$, Daniel Carletti ${ }^{2}$,Tiago M. de Christo ${ }^{2}$, \\ Renan C. Basoni' ${ }^{2}$, Macello D. Queiroz ${ }^{2}$, Igor O. Barbosa ${ }^{2}$, \\ Marcos T. D. Orlando ${ }^{1}$, Jussara F. Fardin ${ }^{2}$ \\ ${ }^{1}$ Departamento de Física, Universidade Federal do Espirito Santo, Vitoria 29075-910 Brasil. \\ ${ }^{2}$ Power Eletronics and Electric Drive Laboratory, Universidade Federal do Espirito Santo, Vitoria 29075-910 Brasil.
}

acarlos.passos@ufes.br

\begin{abstract}
Resumo
Desde o desenvolvimento de materiais de Supercondutores de baixa temperatura crítica (baixa Tc), vários estudos foram publicados em relação a este conceito experimental. Recentemente, os pesquisadores se concentraram no projeto e na aplicação de materiais supercondutores (high-Tc) de alta Tc para desenvolver disjuntores limitadores de corrente de falha. $\mathrm{O}$ funcionamento deste circuito requer grandes razões de corrente prospectiva/limitada, especialmente em áreas perigosas. Apesar disso, vários estudos que descrevem o Limitador de Corrente de Falha Supercondutora (LCFS) que contém membros do bistúrio, mercúrio ou cuprato familiar de ítrio já foram descritos. No entanto, nenhum desses estudos incluiu cupratos de samário. Consequentemente, realizamos um estudo de um pequeno limitador de corrente supercondutor com base em amostras SmBa2Cu3O7-d. Os resultados preliminares indicaram que o cuprato de samário podem ser aplicados em dispositivos de limitação de corrente de falha supercondutores. Em testes utilizando uma amostra policristalina, as propriedades supercondutoras foram mantidas sem modificações na sua estequiometria. Estes resultados sugerem a possibilidade de investigações futuras sobre dispositivos LCFS com base nessas cerâmicas supercondutoras.
\end{abstract}

\begin{abstract}
Since the development of Low Critical Temperature Superconducting (low-Tc) materials, various studies have been published regarding this experimental concept. Recently, researchers have focused on the design and application of high-Tc superconductor (high-Tc) materials to develop fault current limiting circuit breakers. The operation of this circuit requires large prospective/limited current ratios, especially in hazardous areas. In spite of this, several studies describing the Superconducting Fault Current Limiter (SFCL) containing members of the bismuth, mercury or yttrium family cuprate have already been described. However, none of these studies included samarium cuprates. Consequently, we have conducted a study of a small superconducting current limiter device based on $\mathrm{SmBa}_{2} \mathrm{Cu}_{3} \mathrm{O}_{7-\mathrm{d}}$ samples. The preliminary results indicated that samarium cuprates could be applied to build superconducting fault current limiter devices. In tests using a polycrystalline sample, the superconducting properties were retained without modifications to its stoichiometry. These results suggest the possibility of future investigations into SFCL devices based on these superconducting ceramics.
\end{abstract}

Key words: Fault current limiter, High-Tc, ceramics, Sm-123, overcurrent, protection.

\section{Introduction}

A demanda por energia elétrica é crescente e, por causa disso, aumentou o interesse por fontes de energia renováveis (energia verde). Tais fontes de energia o sistema fotovoltaico e os geradores de turbinas eólicos estão atraindo a atenção da comunidade científica devido a sua alta produção e também porque não emitem quaisquer substâncias 
poluidoras [1]. Assim, estas novas fontes de energia estão sendo conectadas aos sistemas de distribuição de baixa tensão, isto é, estão interligados à microrede [1]. No entanto, há um problema crítico nesta configuração porque pode causar um aumento excessivo da corrente de curto-circuito devido à presença das novas fontes de energia na microrede.

Desta forma, é imprescindível desenvolver dispositivos de proteção para aumentar o grau de confiabilidade e de segurança no sistema de microrede [2]. Para reduzir a corrente de falha na rede inteligente com microrede, os LCFS podem ser aplicado porque apresentam uma resposta mais rápida tempo para reduzir a corrente elétrica de curto circuito e também aumentam a estabilidade transitória dos sistemas de energia [3].

Tais dispositivos de proteção contra surtos elétricos vêm recebendo atenção crescente desde a década de 60 e esta tendência deve continuar no futuro. A principal função desses dispositivos é reduzir risco de falha elétrica para que o sistema elétrico seja estável e confiável. Como consequência a área e/ou ambiente onde se encontra o sistema elétrico pode se tornar mais seguro. Dentre eles, o limitador de corrente de falta supercondutor é considerado um dispositivo promissor para reduzir tais correntes elétricas de curto circuito em diversos sistemas elétricos.

Este dispositivo de proteção pode ser dividido em duas categorias básicas: tipo resistivo e tipo indutivo [2]. O tipo resistivo, que será tratado neste presente trabalho, é basicamente um elemento supercondutor em série com circuito a ser protegido. Enquanto que um dispositivo LCFS do tipo indutivo o supercondutor está acoplado magneticamente ao caminho da corrente elétrica de falta, ou seja, baseia-se no efeito Meissner [2].

Existem vários artigos [3-7] que descrevem projetos e desenvolvimentos usando duas cerâmicas supercondutoras de alta temperatura crítica: os compostos $\quad \mathrm{YBa}_{2} \mathrm{Cu}_{3} \mathrm{O}_{7-\mathrm{d}} \quad(\mathrm{Y}-123) \quad[8] \quad \mathrm{e}$
$\mathrm{Bi}_{2} \mathrm{Sr}_{2} \mathrm{CaCu}_{2} \mathrm{O}_{8+y} \quad$ (Bi-2212) [9]. Em geral, os limitadores supercondutores de blocos massivos (bulks) são feito a partir da cerâmica supercondutora Bi-2212. Enquanto que os limitadores com fitas supercondutoras podem ser do composto Y-123 ou do Bi-2212. Contudo, nenhuma proposta publicada incluiu a cerâmica $\mathrm{SmBa}_{2} \mathrm{Cu}_{3} \mathrm{O}_{7-\mathrm{d}}(\mathrm{Sm}-123)$. O composto a base de samário é bastante promissor porque tem a temperatura de transição (Tc) e a densidade de corrente crítica (Jc) similar ao do composto Y-123 [10,11].

Considerando o exposto até aqui, os autores propuseram um dispositivo limitador de corrente de falta supercondutor baseado na cerâmica supercondutora $\mathrm{SmBa}_{2} \mathrm{Cu}_{3} \mathrm{O}_{7-\mathrm{d} \text {. }}$ Nosso principal objetivo aqui é mostrar que composto $\mathrm{Sm}-123$ é capaz de reduz as correntes de falta em circuito elétrico em condições de operação. Além disso, nós investigamos número de vezes que esta cerâmica suporta antes de começar a degradar-se.

\section{Detalhes Experimentais}

\section{Síntese das amostras}

As amostras de $\mathrm{SmBa}_{2} \mathrm{Cu}_{3} \mathrm{O}_{7 \text {-d }}$ foram preparadas pela técnica de reação sólido vapor a partir dos óxidos comerciais $\mathrm{Sm}_{2} \mathrm{O}_{3}$ (99.99\%), $\mathrm{CuO}(99.99 \%)$ e $\mathrm{BaCO}_{3}$ $(99.99 \%)$. Primeiramente, os óxidos foram pesados e misturados em uma atmosfera inerte de argônio (Ar). Depois disso, estes materiais foram homogeneizados em gral de ágata por uma hora. Em seguida, foram colocados em um cadinho de alumina e introduzidos em um forno tubular horizontal cujo patamar foi de $930^{\circ} \mathrm{C}$ por 40 horas em atmosfera comum [10-13]. O precursor obtido foi novamente homogeneizado, prensado no formato cilíndrico (cujo diâmetro foi de $8 \mathrm{~mm}$ ) e recolocado no forno para síntese em $1060^{\circ} \mathrm{C}$ por $5 \mathrm{~min}$ sob fluxo de oxigênio $(10 \mathrm{ml} / \mathrm{min})$. Depois resfriado para patamar de $520^{\circ} \mathrm{C}$ durante 24 horas e finalmente resfriado até a temperatura ambiente [10-13]. 
Após cada tratamento térmico a amostra foi analisada por difração de raios X. Os difratogramas foram obtidos em um aparelho de marca Rigaku, modelo 4053A3, cuja radiação é $\mathrm{CuK} \alpha$ e $\lambda=1,5418$ $\AA$. A amostra foi macerada em gral de ágata e peneirada com granulometria de no máximo $100 \mu \mathrm{m}$ em uma caixa de luva com gás inerte (Ar 99.9\%). Além disso, a amostra foi também caracterizada por microscopia eletrônica de varredura (MEV) ZEISS EVO 40.

\section{Simulação de corrente de falta}

Para fazer o ensaio no dispositivo, uma fonte de corrente ac foi colocada em série com uma resistência elétrica $R_{1}(20$ - $40 \Omega$ ). Como indicado na Figura 1, o elemento supercondutor também estava em série com a fonte de corrente. Para simular de curto circuito ac a chave eletrônica S, controlada por uma porta paralela de um computador PC 486 DX-2, foiacionada de tal forma que uma resistência elétrica $R_{2}(0-20 \Omega)$ era inserida em paralelo a resistência elétrica $R_{1}$. Todos os dados foram coletados por um Osciloscópio Tektronix TDS3014; Amplificador de Ponteira Corrente Tektronix AM503; Ponteira Corrente Tektronix A6302; Ponteira Isoladora Tektronix. As amostras supercondutoras tinham dimensões de $0,24 \times 0,26 \times$ $0,60 \mathrm{~cm}^{3}$ e foram mantidas à temperatura fixa $(77 \mathrm{~K}) \mathrm{e}$ campo magnético aplicado nulo. Os contatos com o supercondutor foram fixados com tinta prata da marca DuPont e apresentaram resistência média de $0,10 \Omega$. Todos os testes foram realizados com frequência da rede local $(60 \mathrm{~Hz})$.

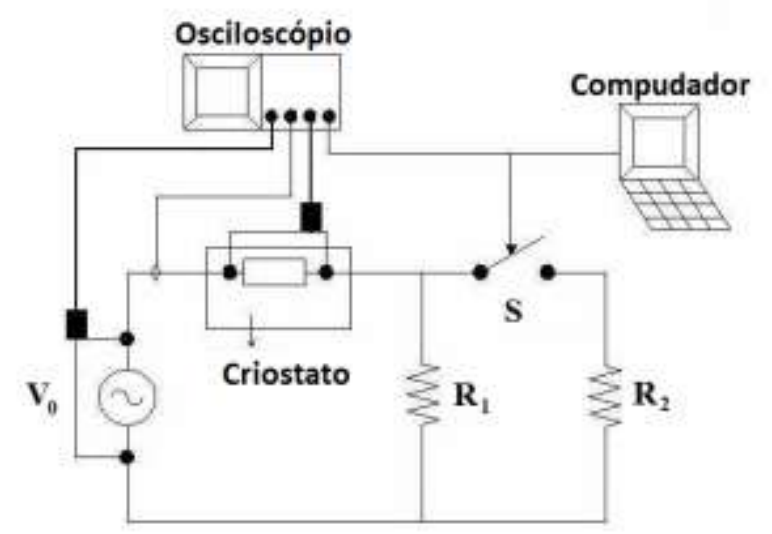

Fig. 1: Esquema da bancada para realizar o ensaio de curto circuito. A cerâmica é inserida no criostato e mantida em 77K.

\section{Resultados e Discussões}

\subsection{Caracterização da amostra}

Os espectros foram medidos a partir de $5^{\circ}$ a $80^{\circ} \mathrm{com}$ um tamanho de passo de $0.02^{\circ}$, e os tempos de medida variou de 15 a $25 \mathrm{~s}$. Um espectro típico é mostrado na figura 2. A fase principal encontrada foi do $\mathrm{Sm}-123$, além disso, os resíduos de $\mathrm{Sm}_{2} \mathrm{O}_{3}$ e $\mathrm{BaCuO}_{2+\mathrm{x}}$ foram também encontrados.

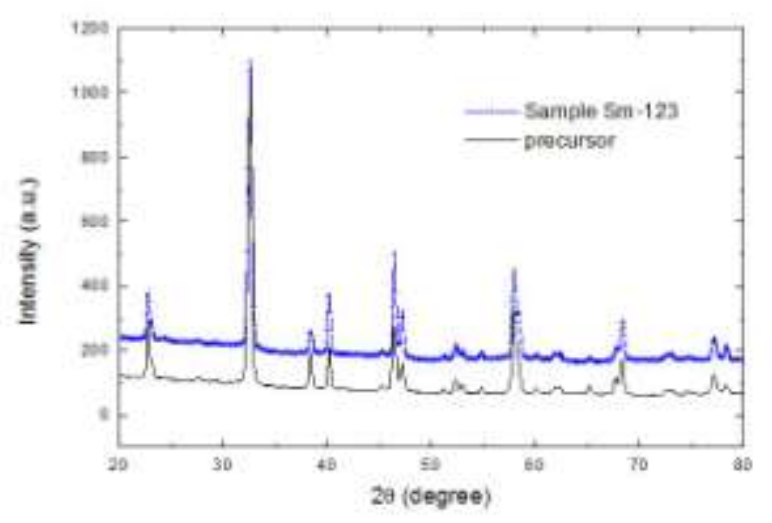

Figure 2: XRD patterns ( $\mathrm{Cu} \mathrm{K} \alpha$ radiation) of the $\mathrm{Sm}-123$ ceramics. 
A figura 3 mostra a imagem obtida por microscopia eletrônica de varredura. Nesta figura pode ser observada a dispersão de tamanho de grãos e precipitados. Com base nas análises de espectrometria de dispersão de energia (EDS) foi possível obter as concentrações de $\mathrm{Sm}, \mathrm{Ba}, \mathrm{Cu}$ e $\mathrm{O}$. As análises de EDS foram feitas de forma global, no contorno de grão e no centro de grão Os resultados da análise estão dispostos na Tabela 1.

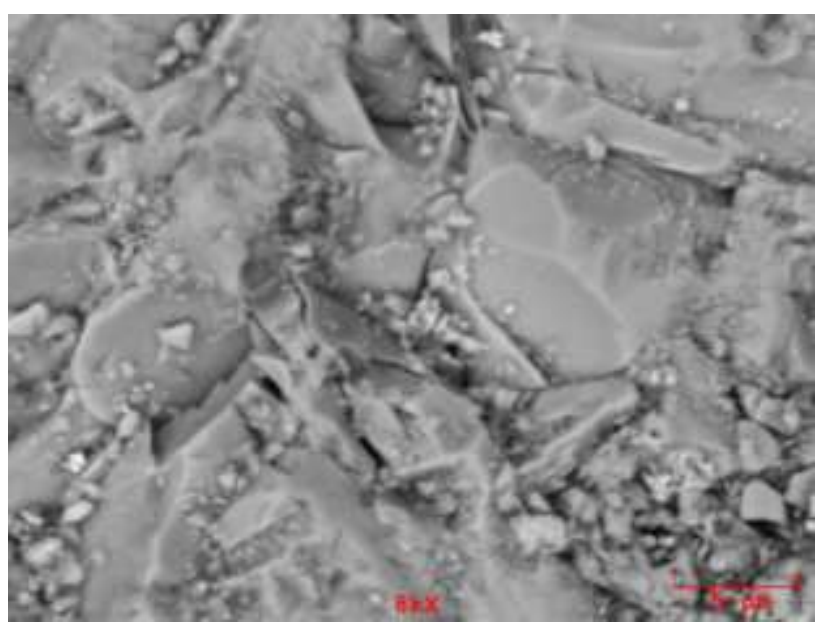

Figura 3: Micrografia da cerâmica $\mathrm{SmBa}_{2} \mathrm{Cu}_{3} \mathrm{O}_{7-\mathrm{d}}$ ampliado 8 mil vezes.

Tabela 1: Análise do centro e o contorno do grão das amostras medidas por EDS

\begin{tabular}{|llll|}
\hline Elemento & $\begin{array}{l}\text { Concentração } \\
\text { do centro de } \\
\text { grão }(w t \%)\end{array}$ & $\begin{array}{l}\text { Concentração } \\
\text { da borda de } \\
\text { grão }(w t \%)\end{array}$ & $\begin{array}{l}\text { Concentração } \\
\text { Olobal (wt \%) }\end{array}$ \\
\hline $\mathrm{Cu}$ & 12,478 & 11,867 & 11.456 \\
$\mathrm{Ba}$ & 14,239 & 14,820 & 13,689 \\
& 49,822 & 50,471 & 51,22 \\
$\mathrm{Sm}$ & 23,461 & 22,842 & 23,625 \\
\hline
\end{tabular}

Estes resultados mostraram que o íon de $\mathrm{Ba}$ tem excesso de concentração. Este resultado sugere que nem todo bário da estequiometria inicial não reagiu durante a síntese. Isto justifica porque foram encontrado composições de $\mathrm{BaCuO}_{2+\mathrm{x}}$ e $\mathrm{Sm}_{2} \mathrm{O}_{3}$ na análise de difração raios X. Estas fases residuais são centros espalhadores naturais de corrente elétrica.

\subsection{Dispositivo limitador de corrente - SFCL}

Como primeiro teste do limitador de corrente elétrica, um fio de ouro com diâmetro de $2 \mathrm{~mm}$ foi colocado em série com a resistência elétrica $R_{1}(36,2 \Omega)$ e o conjunto estava submetida a $10,0 \mathrm{~V}_{\text {pico }}$ tal que a corrente elétrica foi de $\mathrm{I}=0,25 \mathrm{~A}_{\text {pico. }}$. Quando a chave foi acionada $(\mathrm{t}=0 \mathrm{~s})$, uma segunda resistência elétrica $\left(R_{2}=1,1 \Omega\right)$ ficou em paralelo a $R_{1}$ com a finalidade de reduzir a impedância do circuito elétrico tal que a corrente esperada foi de $\mathrm{I}=8,2 \mathrm{~A}_{\text {pico. }}$ A figura 4 mostra a forma de onda da corrente durante este teste.

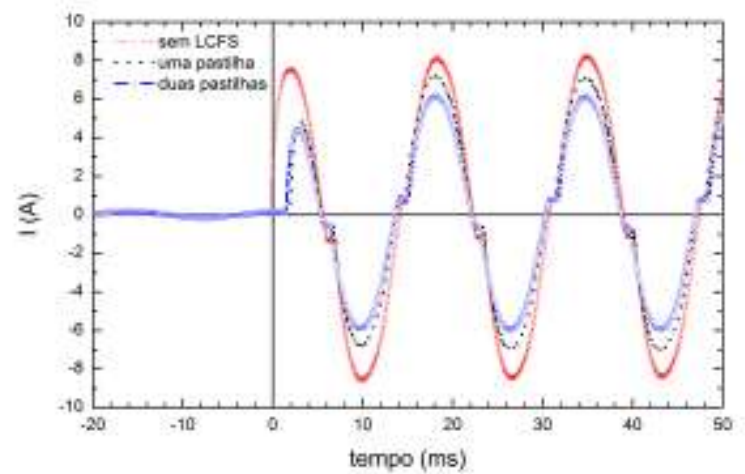

Figura 4: A formas de onda da corrente para o segundo e o terceiro testes. A curva pontilha é o teste com uma pastilha de Sm-123 e curva símbolo circular representa o teste com dois elementos supercondutores ligados em série.

O mesmo procedimento foi repetido para o segundo teste, porém o elemento supercondutor Sm-123 estava em série com $R_{1}$. É importante ressaltar que resistência de contatos foi de $0,94 \Omega$ à temperatura ambiente. Nesta nova configuração, o elemento 
supercondutor reduziu a corrente elétrica durante o intervalo de tempo que a chave foi fechada. A corrente de pico limitada foi 7,2 $\mathrm{A}_{\text {pico }}$ conforme figura 4 . Desta forma a razão entre a de corrente esperada e a limitada foi $\sim 1.1$, levando-se em consideração que a amostra tem espessura de $0,24 \mathrm{~cm}$. Quanto maior esta razão menor os problemas de estresses mecânicos e elétricos durante um curto-circuito, ou seja, reduzir a corrente de falha minimiza os problemas.

Um ciclo completo com fechamento e abertura da chave eletrônica foi realizado com a finalidade de se verificar se a pastilha de Sm-123 retorna ao estado supercondutor imediatamente após a retirada do curto circuito. Conforme mostrado na figura 5, as condições normais de operação do circuito elétrico são reestabelecidas. Este resultado indica que a cerâmica mantém sua propriedade supercondutora.

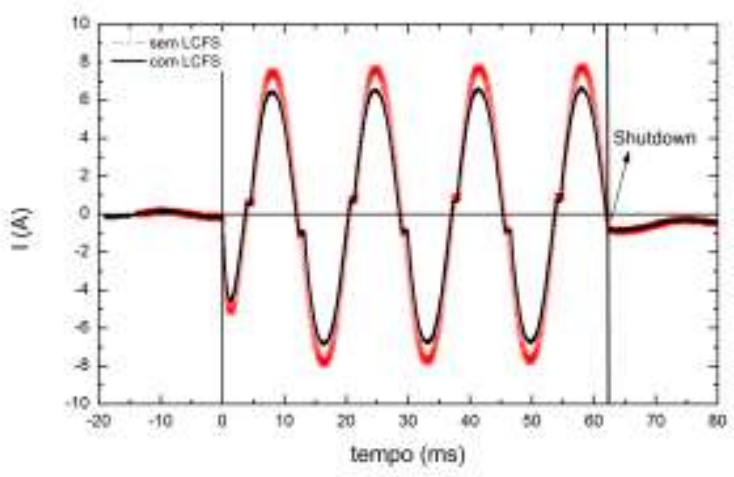

Figura 5: A formas de onda da corrente durante do segundo teste tomando o ciclo completo, fechamento e abertura da chave eletrônica.

$\mathrm{O}$ quarto teste foi realizado com duas patilhas de Sm-123 ligadas em série e com as mesma configurações do circuito elétrico descritas anteriormente. Neste caso, pode-se observar que os elementos supercondutores reduziram a corrente elétrica de curto circuito, $I=6,1 \mathrm{~A}_{\text {pico }}$ (veja a figura 4) cuja razão entre a corrente esperada e a limitada foi aproximadamente de 1.3.

Durante o evento de falta, o elemento supercondutor passa para o estado normal. Neste momento, a corrente de falha é limitada pela resistência da amostra e ocorre dissipação de energia (aquecimento nas bordas dos grãos). Esta corrente elétrica percola os grãos da cerâmica e isto pode danificar a região intergranular. Os aquecimentos nas regiões de contorno do grão dependem do intervalo de tempo necessário para retornar ao estado supercondutor (tempo de persistência do curto circuito) e número de vezes que o curto circuito acontece. Para verificar esta possibilidade foram feitos outros seis testes com as mesmas configurações de associação de resistores do segundo teste.

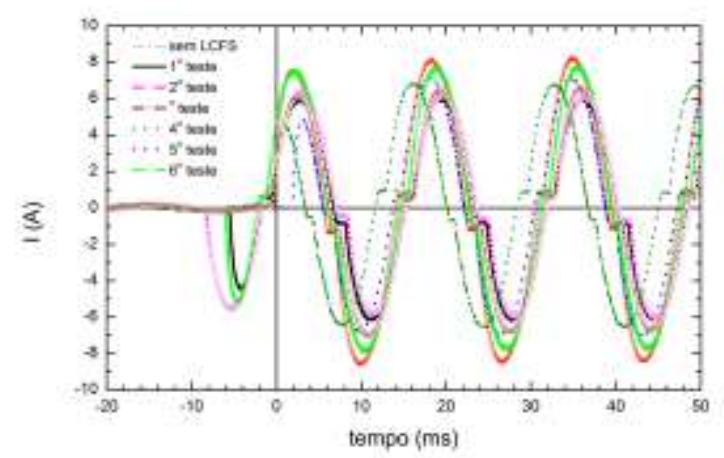

Figura 6: A formas de onda da corrente para diversos testes com a cerâmica Sm-123.

A figura 6 apresenta os resultados de oito estes de curto circuito. É possível perceber que há limitação da corrente de falta, mas o desempenho do dispositivo diminuiu após quarto ensaio. Em nossa opinião a circuito, limite de reversibilidade do dispositivo LCFS é quatro vezes. Acima deste número de ensaios a amostra cerâmica degradou, ou seja, os contornos de grãos não são mais capazes de conduzir corrente elétrica acima do valor da corrente crítica da amostra. 


\section{Conclusões}

Este trabalho mostrou um estudo preliminar sobre o dispositivo limitador de corrente supercondutor do tipo resistivo usando a cerâmica $\mathrm{SmBa}_{2} \mathrm{Cu}_{3} \mathrm{O}_{7-\text { d. }}$. Os testes confirmaram a capacidade do Sm-123 limitar correntes elétricas de falha. A amostra supercondutora não apresentou qualquer degradação para o limite de quatro ensaios de falha elétrica, conforme verificado no teste de recuperação. No entanto a partir deste número de eventos de falha elétrica, a amostra perdeu gradativamente as propriedades supercondutoras e, por consequência, tornou-se ineficiente para limitar corrente de curto circuito. Estes resultados serão aplicados para determinar as condições ideais em futuros dispositivos supercondutores limitadores de corrente da cerâmica Sm-123.

\section{Acknowledgments}

Os autores gostariam de agradecer o financiamento das agências de fomento FAPES \#67666027.

\section{References}

[1] F. L. da Silva, Análise do desempenho de uma microrrede com múltiplas unidades de geração distribuída. Dissertação apresentada ao Programa de PósGraduação em Engenharia Elétrica do Centro Tecnológico da Universidade Federal do Espírito Santo 2011.

[2] Feng Zheng, Changhong Deng, Lei Chen, Member, IEEE, Shichun Li, Yang Liu, and Yuxiang Liao, Transient Performance Improvement of Microgrid by a Resistive Superconducting Fault Current Limiter. IEEE Transactions On Applied Superconductivity, 25 (3) (2015) 5602305-1 - 5602305-5.

[3] W. T. B. de Sousa, A Polasek, R. Dias, R. de Andrade Jr., Limitadores de corrente de curto-circuito supercondutores: principais conceitos e testes. Revista Brasileira de Ensino de Física, 34(4) (2012) 4313-1 4313-8.

[4] R. B. Standeler, Protection of Elecronic Circuits From Overvoltages, (Ed.) Dover Science Books, Mineola, 2002, pp. 3-4.
[5] E.M. Leung, A. Rodriguez, G.W. Albert, B. Burley, M. Dew, P. Gurrola, et al., High temperature superconducting fault current limiter development, IEEE Trans. on Applied Superconductivity 7 (2) (1997) 985-988.

[6] W. Paul, M. Chen, M. Lakner, D. Braun, W. Lanz, Fault current limiter based on high temperature superconductors - different concepts, test results, simulations, applications, Physica C, 354 (1-4) (2001) 27-33.

[7] S.-H. Lim, H.-S. Choi, and B.-S. Han, Fault current limiting characteristics due to winding direction between coil 1 and coil 2 in a flux-lock type SFCL Physica C 416 (1-2) (2004) 34-42.

[8] C. A. Baldan, C. Y. Shigue, D. G. Pinatti, E. Ruppert Filho, R. C. Freitas, and R. P. Homrich, Resistive fault current limiter using hts single-layer coils, Physica C 408-410 (1-4) (2004) 937-939.

[9] M. Chen, L. Donzel, M. Lakner, and W. Paul, High temperature superconductors for power applications, J. Eur. Ceram. Soc. 24 (6) (2004), 1815-1822.

[10] M. R. Osorio, L. Cabo, J. A. Vieira, and F. Vidal, Non-homogeneous quench of the superconducting secondary of an inductive fault current limiter: implications for current limitation Supercond. Sci. Technol. 17 (7) (2004) 868-875.

[11] N. T. Nguyen and P. Tixador, A YBCO-coated conductor for a fault current limiter: architecture influences and optical study, Supercond. Sci. Technol. 23 (2) (2010) 025008-1 - 025008-12.

[12] C. J. Hawley, F. Darmann and T. P. Beales, Performance of a $1 \mathrm{MV}$ A high temperature superconductors-enabled saturable magnetic core-type fault current limiter, Supercond. Sci. Technol. 18 (3) (2005) 255-259.

[13] T. Iwasaki, , H. Ikuta, M. Yoshikawa, Y. Yanagi, Y. Itoh, U. Mizutani, Annealing melt-processed Sm-Ba-Cu-O bulk superconductors under 10 atm oxygen, Physica C 412-414 (2004) 580-585

[14] N. Hari Babu, K. Iida, Y. Shi, D.A. Cardwell, Processing of bulk $\mathrm{Sm}-\mathrm{Ba}-\mathrm{Cu}-\mathrm{O}$ nano-composite superconductors, Physica C 468 (2008) 1340-1344.

[15] C. A. C. Passos, M. T. Orlando, J. L. Passamai, E. F. Medeiros, F. D. Oliveira, J.F. Fardin, D. S. L. Simonetti, Superconducting fault current limiter device based on Hg,Re-1223 superconductor, Applied Physics Letters 89 (2006), 2425031-2425033.

[16] J. Maňka, A. Cigáň, J. Polovková, A. Koňakovský, A. Prnová, Effects of Slight Non-Stoichiometry in $\mathrm{Sm}-\mathrm{Ba}-\mathrm{Cu}-\mathrm{O}$ Systems on Superconducting Characteristics, Measurement Science Review, 11 (1) (2011) 9-14 